\title{
Surface-enhanced chiroptical spectroscopy with superchiral surface waves
}

\author{
Giovanni Pellegrini, ${ }^{[a]}$ Marco Finazzi, ${ }^{[a]}$ Michele Celebrano, ${ }^{[a]}$ Lamberto Duò, ${ }^{[a]}$ and Paolo \\ Biagioni*[a]
}

\begin{abstract}
We study the chiroptical properties of one-dimensional photonic crystals supporting superchiral surface waves by introducing a simple formalism based on the Fresnel reflection matrix. We show that the proposed framework provides useful insights on the behaviour of all the relevant chiroptical quantities, allowing for a deeper understanding of surface-enhanced chiral sensing platforms based on one-dimensional photonic crystals. Finally we analyze and discuss the limitations of such platforms as the surface concentration of the target chiral analytes is gradually increased.
\end{abstract}

Keywords: Optical chirality, Superchirality, Surface-enhanced sensing, Photonic crystals

\section{Introduction}

The analysis and separation of chiral molecules has lately gained traction in the pharmaceutical and biochemical industry. ${ }^{1,2}$ The majority of new drugs developed by the pharmaceutical industry are chiral and it is expected that nearly $95 \%$ of drugs would be chiral by $2020 .^{3,4}$ Circular dichroism (CD) spectroscopy, i.e., the measurement of the differential absorption between left $(\mathrm{L})$ and right $(\mathrm{R})$ circularly polarized light $(\mathrm{CPL})$, is one of the most relevant tools to quantify the enantiomeric excess and determine the conformation and configuration of chiral molecules. However, the differential nature of $C D$ signals usually leads to extremely weak signal-tonoise ratios, making the analysis of small amounts of chiral analytes extremely challenging. . $^{1,2,5,6}$

In this context, novel approaches have been proposed over the last few years to enhance the CD signal by tailoring the chiral properties of the probing electromagnetic field through the control of the associated optical chirality $C$, which is defined as: ${ }^{5-9}$

$$
C=-\frac{\varepsilon_{0} \omega}{2} \operatorname{Im}\left(\mathbf{E}^{*} \cdot \mathbf{B}\right)
$$

This quantity is a time-even pseudoscalar, i.e., it behaves as a chiral object under mirror inversion, and is one of the factors determining the degree of asymmetry in the absorption rate of a chiral molecule between $L$ and $R$ circularly-polarized light in the dipolar approximation. Circularly-polarized plane waves are a common example of a chiral electromagnetic field, with their optical chirality for a unitary intensity wave expressed as $C_{\mathrm{CPL}}^{\mathrm{L}, \mathrm{R}}=$ $\pm \varepsilon_{0} \omega / 2 c .^{5}$ Likewise, a field distribution where $C$ is beyond the $C_{\mathrm{CPL}}^{\mathrm{L}, \mathrm{R}}$ value is dubbed 'superchiral'. In this framework, an ideal chiral sensing platform is required to (i) provide uniform superchiral optical fields $\left(|C| \gg\left|C_{\mathrm{CPL}}^{\mathrm{L}, \mathrm{R}}\right|\right)$ over large surfaces, (ii) generate optical chiralities of both handednesses upon reversing the polarization state of the incident field, (iii) work at wavelengths ranging from the near-UV to the IR depending on the platform design, with a particular attention to the high-energy range of the spectrum, below $400 \mathrm{~nm}$, where most electronic molecular transitions occur, ${ }^{7,9,10}$ and (iv) allow for the faithful reconstruction of the intrinsic line-shape of a specific $C D$ resonance.

Plasmonic nanostructures have been widely proposed and employed as a viable solution for the enhancement of the optical chiral response of biomolecules..$^{2,7-20}$ However, despite the recent results, to date chiral plasmonic sensing does not meet all the above criteria simultaneously, since superchiral plasmonic fields are spatially confined to the so-called 'hot spots' and large homogeneous optical chiralities are usually obtained only for chiral plasmonic nanostructures, thus hindering the possibility of handedness switching upon reversal of the incident polarization state. Moreover, although novel plasmonic materials such as aluminum can operate in the blue and near-UV spectral range, ${ }^{21}$ most noble metals suffer from large interband absorption at such wavelengths. Finally, the coupling between the electronic molecular resonance and the plasmonic resonance usually results in Fano-like spectral features that cannot be directly compared to standard databases for electronic $C D$.

Recently, we introduced a radically different paradigm, which exploits the optical properties of one-dimensional photonic crystals (1DPCs) to generate superchiral electromagnetic fields. ${ }^{22-24}$ The whole approach rests on the idea that 1DPCs support both transverse-electric (TE) and transverse-magnetic (TM) Bloch surface waves (BSWs). An appropriate engineering of the multilayer structure, specifically the introduction of an

[a] Dipartimento di Fisica Politecnico di Milano Piazza Leonardo da Vinci 32, 20133, Milano, Italy

\section{[*] Paolo Biagioni}

Dipartimento di Fisica

Politecnico di Milano

Piazza Leonardo da Vinci 32, 20133, Milano, Italy E-mail: paolo.biagioni@polimi.it

Received: ((will be filled in by the editorial staff))

Revised: ((will be filled in by the editorial staff)) Published online: ((will be filled in by the editorial staff)) 
(a)

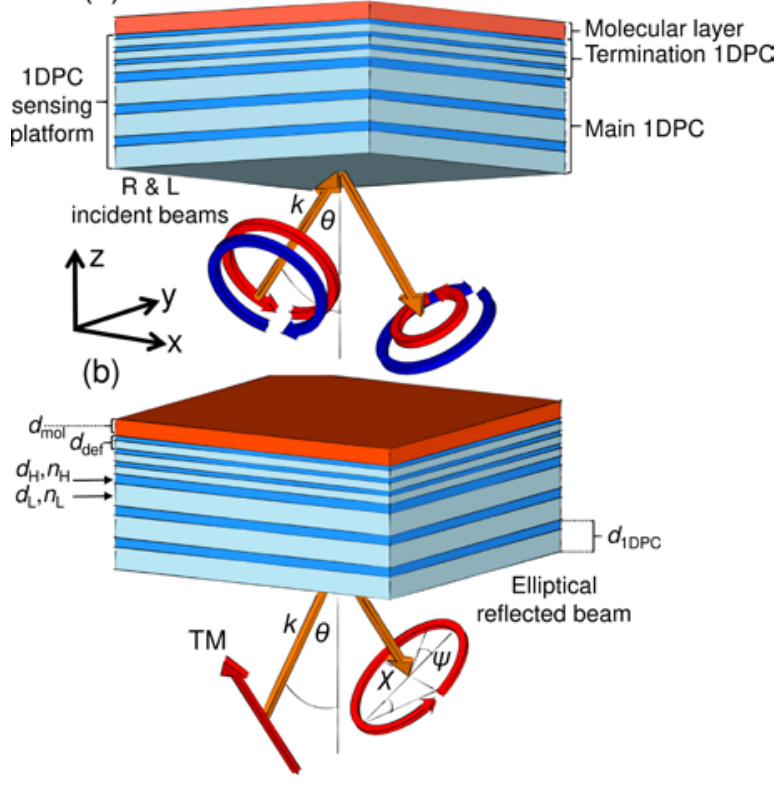

FIGURE 1 (a) A schematic representation of the 1DPC platform. The periodicities of the main $1 D P C$ and the additional $1 D P C$ termination are defined as $d_{1 \mathrm{DPC}}$ and $d_{\text {def }}$, respectively, while $d_{\mathrm{H}, \mathrm{L}}$ stands for the thickness of the high and low refractive index materials in the main 1DPC. The thickness of the optically active molecular layer is defined as $d_{\text {mol }}$. (b) Schematic representation with TM illumination for an ellipticity and rotation measurement.

additional 1DPC termination with a much shorter lattice parameter (Figure 1(a)), allows for the superposition of the TE and TM dispersion relations. This solution admits the simultaneous excitation of TE and TM surface waves, and therefore the excitation of superchiral surface waves at the 1DPC sensing platform surface, much like the combination of two orthogonal linear polarization states with the appropriate $\pi / 2$ phase shift would result in a circular polarization state in free space (Figure 2). ${ }^{22}$ This configuration leads to superchiral surface waves that provide homogeneous, superchiral and switchable local fields over arbitrarily large surfaces and a wide spectral range. Optical chirality enhancements are predicted to be well above 1 order of magnitude and CD signal enhancements exceeds 2 orders of magnitude, thus paving the road to fluorescence-free surface enhanced CD sensing and spectroscopy on extremely low analyte amounts. ${ }^{22}$

To better clarify the chiroptical properties of 1DPC sensing platforms, we introduce here a simple formalism where all the relevant chiroptical quantities are expressed in terms of the Fresnel reflection matrix for the system (1DPC sensing platform + chiral molecules). We obtain the reflection matrix by using the transfer matrix approach together with the constitutive relations for the chiral medium. We then proceed to illustrate how the chiroptical properties of the multilayer structure are strongly related to the simultaneous excitation of TE and TM BSWs, and finally discuss the $C D, \chi$ and $\psi$ enhancement properties along with some existing limitations.

\section{Materials and Methods}

A typical model system consists of a finite 1DPC with alternating high $\left(n_{\mathrm{H}}\right)$ and low $\left(n_{\mathrm{L}}\right)$ refractive index materials (the 1DPC sensing platform) and an optically active thin layer at its

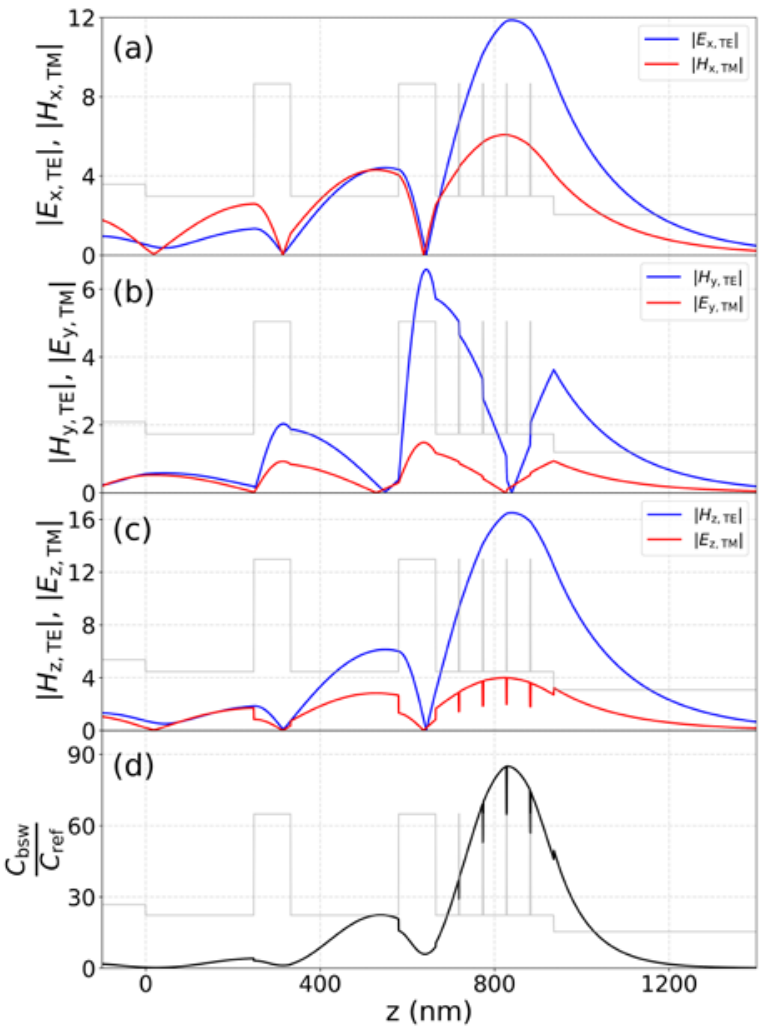

FIGURE 2 Local field and optical chirality plots along the $z$-axis, across the 1DPC sensing platform, for TE and TM incident plane waves. Plane waves are incident from the left. (a) $E_{x}$ and $H_{x}$ components. (b) $H_{y}$ and $E_{y}$ components. (c) $H_{z}$ and $E_{z}$ components. (d) Optical chirality enhancement for an elliptically polarized (as in Equation 8) incident plane wave.

termination (the measured sample), as illustrated in Figure 1. We study the system by solving the multilayer transfer matrix problem including the constitutive relations for the chiral medium: ${ }^{25}$

$$
\begin{aligned}
& \mathbf{D}=\varepsilon_{\mathrm{c}} \varepsilon_{0} \mathbf{E}+i \xi_{\mathrm{c}} \mathbf{B}, \\
& \mathbf{H}=\frac{\mathbf{B}}{\mu \mu 0}+i \xi_{\mathrm{c}} \mathbf{E}
\end{aligned}
$$

where $\varepsilon_{\mathrm{c}}$ and $\mu$ denote the relative permittivity and permeability, while $\xi_{\mathrm{c}}$ is the chiral optical admittance, i.e. the parameter that controls the chirality strength. We work with a non-magnetic medium ( $\mu=1)$ and we define its optical parameters as: ${ }^{12}$

$$
\begin{aligned}
& \varepsilon_{\mathrm{c}}=\varepsilon_{\mathrm{c} 0}-\gamma_{\mathrm{c}}\left(\frac{1}{\hbar \omega-\hbar \omega_{0}+i \Gamma}-\frac{1}{\hbar \omega+\hbar \omega_{0}+i \Gamma}\right), \\
& \xi_{\mathrm{c}}=\beta_{\mathrm{c}}\left(\frac{1}{\hbar \omega+\hbar \omega_{0}+i \Gamma}+\frac{1}{\hbar \omega-\hbar \omega_{0}+i \Gamma}\right),
\end{aligned}
$$

where the coefficients $\gamma_{\mathrm{c}}$ and $\beta_{\mathrm{c}}$ determine the magnitude of the molecular absorption and of the chiral optical constant, $\hbar \omega_{0}$ is the energy of the electronic transition, $\Gamma$ is the damping and $\varepsilon_{\mathrm{c} 0}$ is the background optical constant, i.e. the optical constant of the molecular material away from the electronic resonance.

In the following, we assume that the chiral medium deposited on top of our 1DPC sensing platform is uniform and isotropic: chiral molecules, either suspended in a solution flowing into a microfluidic channel or embedded into an amorphous film spin- 
coated on top of the 1DPC termination are practical examples of the modeled configuration.

The reflection optical properties of the system can be entirely described by the Fresnel reflection matrix. The overall stack displays a cylindrical symmetry around the $z$-axis, i.e. around the normal to the sample surface, which, together with the presence of the optically active molecular layer, leads to a Fresnel matrix defined as: ${ }^{26}$

$$
\mathcal{R}=\left(\begin{array}{cc}
r_{T M, T M} & r_{T M, T E} \\
-r_{T M, T E} & r_{T E, T E}
\end{array}\right)
$$

The off-diagonal element $r_{T M, T E}$ is different from zero only in the presence of optical activity, thus allowing to define without ambiguity all the chiroptical quantities in terms of the Fresnel matrix coefficients. ${ }^{26}$ If we then define the incident polarization state with the Jones vector $\left(a_{i n}, b_{i n}\right)$ the reflected polarization state becomes:

$$
\left(\begin{array}{l}
a_{\text {out }} \\
b_{\text {out }}
\end{array}\right)=\mathcal{R}\left(\begin{array}{l}
a_{\text {in }} \\
b_{\text {in }}
\end{array}\right)
$$

Finally, once the reflected polarization states are known, all the relevant chiroptical quantities can be easily computed. In order to obtain right and left superchiral surface waves, the incident polarization states are defined by the Jones vectors $\left(a_{i n}, b_{i n}\right)_{+}=$ $\left(1, \pm e^{i \phi}\right)$, where $\phi=\pi / 2-\Delta \phi_{\mathrm{TE}, \mathrm{TM}}$ and $\Delta \phi_{\mathrm{TE}, \mathrm{TM}}$ is the relative phase delay introduced by the 1DPC. ${ }^{22}$ The incident polarization states represent left and right elliptical plane waves, in substitution of the circular ones, to compensate for the phase shift introduced by the presence of the 1DPC. The polarization states after reflection can therefore be written as

$$
\left(\begin{array}{l}
a_{\text {out }} \\
b_{\text {out }}
\end{array}\right)_{ \pm}=\mathcal{R}\left(\begin{array}{c}
1 \\
\pm e^{i \phi}
\end{array}\right)
$$

In order to compare our signal with a standard CD signal, we calculate a reflectivity dichroism defined as $R D=\log _{10}\left(R_{L} / R_{R}\right)$ with $R_{L}\left(R_{R}\right)$ the left (right) reflected intensities:

$$
R D \simeq \frac{4}{\log (10)} \frac{\operatorname{Re}\left\{e^{-i \phi}\left(r_{T M, T M} r_{T M, T E}^{*}-r_{T M, T E} r_{T E, T E}^{*}\right)\right\}}{\left|r_{T M, T M}\right|^{2}+\left|r_{T E, T E}\right|^{2}},
$$

where we have made use of the fact that $\left|r_{T E, T M}\right| \ll\left|r_{T M, T M}\right|$ and $\left|r_{T E, T M}\right| \ll\left|r_{T E, T E}\right|$ (it should be noted here that the exact and approximated $R D$ calculations differ by less than $10^{-7} \mathrm{mdeg}$ ). The reflectivity dichroism obtained from the sensing platform must be compared to a reference CD spectrum. To do so we compute the CD spectrum for the same optically active thin film on a $n=1.45$ glass substrate for normal illumination with a circularly polarized plane wave of unitary intensity. We then define the reference signal as $C D_{\text {ref }}=A_{\text {ref }}^{\mathrm{R}}-A_{\text {ref }}^{\mathrm{L}}$, where $A_{\text {ref }}^{\mathrm{R}, \mathrm{L}}=-\log _{10}\left(T^{\mathrm{R}, \mathrm{L}}\right)$ and $T^{\mathrm{R}, \mathrm{L}}$ is the computed transmittance.

We perform a similar procedure to obtain the expressions for the ellipticity $\chi$ and the rotation $\psi$ as a function of $\mathcal{R}$. In this case the incident Jones vectors are defined as $\left(a_{i n}, b_{i n}\right)_{T M}=(1,0)$ and $\left(a_{i n}, b_{i n}\right)_{T E}=(0,1)$ for TM and TE polarizations, respectively, with the reflected fields thus becoming $\left(a_{\text {out }}, b_{\text {out }}\right)_{T M}=$ $\left(r_{T M, T M},-r_{T M, T E}\right)$ and $\left(a_{\text {out }}, b_{\text {out }}\right)_{T E}=\left(r_{T M, T E}, r_{T M, T E}\right)$. If we introduce the Stokes parameters for the reflected light as

$$
\begin{aligned}
& S_{0}=a_{\text {out }} a_{\text {out }}^{*}+b_{\text {out }} b_{\text {out }}^{*}, \\
& S_{1}=a_{\text {out }} a_{\text {out }}^{*}-b_{\text {out }} b_{\text {out }}^{*}, \\
& S_{2}=a_{\text {out }} b_{\text {out }}^{*}+b_{\text {out }} a_{\text {out }}^{*}, \\
& S_{3}=i\left(a_{\text {out }} b_{\text {out }}^{*}-b_{\text {out }} a_{\text {out }}^{*}\right),
\end{aligned}
$$

the ellipticity and the rotation, as illustrated in Figure 1, may be finally expressed as:

$$
\begin{aligned}
& \chi=\frac{1}{2} \arctan \left(\frac{S_{2}}{S_{1}}\right), \\
& \psi=\frac{1}{2} \arcsin \left(\frac{S_{3}}{S_{0}}\right) .
\end{aligned}
$$

The expressions above may be further simplified recalling that the off-diagonal terms of $\mathcal{R}$ are much smaller than the diagonal ones, to eventually obtain the ellipticity

$$
\begin{gathered}
\chi_{T M}=-\frac{\operatorname{Im}\left\{r_{T M, T E} r_{T M, T M}^{*}\right\}}{\left|r_{T M, T M}\right|^{2}}, \\
\chi_{T E}=-\frac{\operatorname{Im}\left\{r_{T M, T E} r_{T E, T E}^{*}\right\}}{\left|r_{T E, T E}\right|^{2}},
\end{gathered}
$$

and the rotation

$$
\begin{gathered}
\psi_{T M}=-\frac{\operatorname{Re}\left\{r_{T M, T M} r_{T M, T E}^{*}\right\}}{\left|r_{T M, T M}\right|^{2}}, \\
\psi_{T E}=-\frac{\operatorname{Re}\left\{r_{T E, T E} r_{T M, T E}^{*}\right\}}{\left|r_{T E, T E}\right|^{2}} .
\end{gathered}
$$
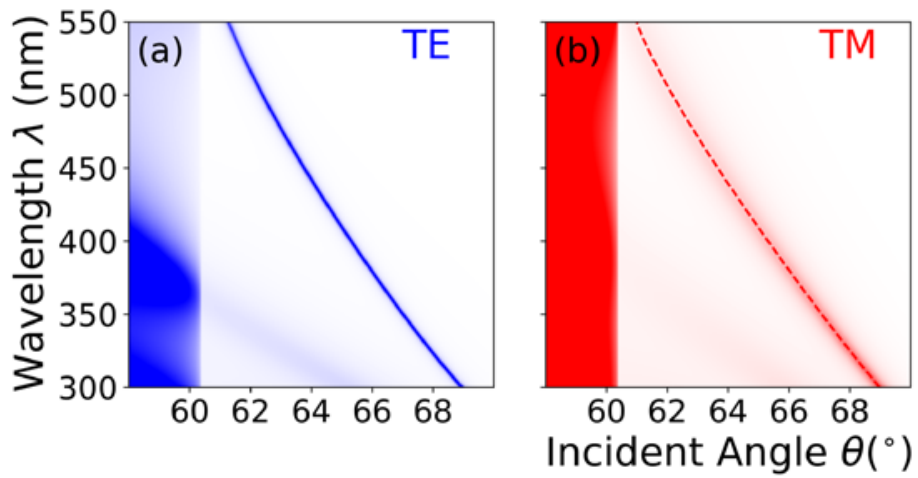

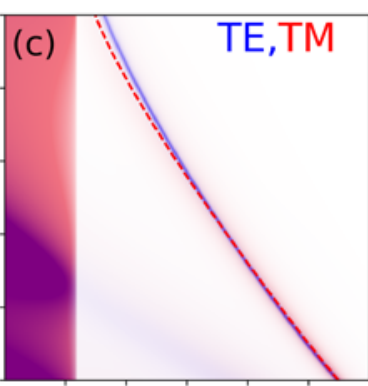

$60 \quad 62 \quad 64 \quad 66 \quad 68$

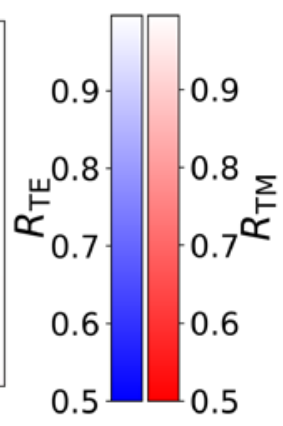

FIGURE 3 Reflectance density plots for TE and TM illuminations, and their superposition. The surface modes are visible as a thin, dark blue, diagonal band (TE) and a lighter and broader red halo (TM). The thin dashed line serves as a guide to the eye to track the TM surface mode minimum along the $(\lambda, \theta)$ plane. 


\section{Results and Discussion}

The expressions introduced above clearly show that the chiroptical signals are directly proportional to the off-diagonal term $r_{T M, T E}$ of the Fresnel reflection matrix. Equally interesting is the fact that all the expressions diverge when the absolute values of the diagonal terms $\left|r_{T M, T M}\right|$ and $\left|r_{T E, T E}\right|$ tend to zero. The divergence actually stems from the neglection of the $\left|r_{T M, T E}\right|$ terms at the denominator, nevertheless this is a strong indication that, in principle, properly designed 1DPCs can efficiently remove the achiral background from the measured chiroptical signals, somewhat in analogy with previous approaches. ${ }^{5,6}$ It is finally interesting to note that, if $r_{T M, T M}=r_{T E, T E}$, our expressions for $R D$, $\chi$ and $\psi$ reduce to the traditional ones for molecules in isotropic environments (i.e. in solution), with the usual $R D$ proportionality to the ellipticity restored to $R D \simeq \chi(\log (10) / 4)$ as in a standard $C D$ signal.

To better illustrate the chiroptical properties of 1DPCs supporting simultaneous TE and TM surface waves, we model the multilayer already described in Ref. 22 and schematically depicted in Figure 1. For our structure we choose $\mathrm{Ta}_{2} \mathrm{O}_{5}\left(n_{\mathrm{H}}=2.06+\right.$ $0.001 i)$ and $\mathrm{SiO}_{2}\left(n_{\mathrm{L}}=1.454+0.0001 i\right)$, while the upper semiinfinite space is water $\left(n_{\text {water }}=1.33\right)$ and the incident medium is a BK7 glass $\left(n_{\text {inc }}=1.53\right) .{ }^{24}$ The crystal periodicity is $d_{1 \mathrm{DPC}}=333$ $\mathrm{nm}$, and the respective layer thicknesses are $d_{\mathrm{H}}=f_{\mathrm{H}} d_{1 \mathrm{DPC}}$ and $d_{\mathrm{L}}=\left(1-f_{\mathrm{H}}\right) d_{1 \mathrm{DPC}}$, where $f_{\mathrm{H}}=0.26$ is the filling factor. To achieve the superposition of TE and TM dispersion relations we design a termination 1DPC characterized by a periodicity $d_{\text {def }}$ much smaller than $d_{1 \mathrm{DPC}}$. The additional multilayer consists of $N_{\text {def }}=5$ periods of alternating $\mathrm{Ta}_{2} \mathrm{O}_{5}$ and $\mathrm{SiO}_{2}$ layers. The total thickness is expressed as a function of the main 1DPC parameters as $t_{\mathrm{def}}=d_{\mathrm{L}} c_{\mathrm{def}}$, with $c_{\mathrm{def}}=1.1$. The periodicity of the 1DPC termination then becomes $d_{\mathrm{def}}=t_{\mathrm{def}} / N_{\mathrm{def}}$, and accordingly the layer thicknesses are $d_{\mathrm{H}, \mathrm{def}}=f_{\mathrm{H}, \mathrm{def}} d_{\mathrm{def}}$ and $d_{\mathrm{L}, \mathrm{def}}=(1-$ $\left.f_{\mathrm{H}, \mathrm{def}}\right) d_{\text {def }}$, where $f_{\mathrm{H}, \mathrm{def}}=0.03$ is the terminal 1DPC filling factor. The additional 1DPC is terminated with a low-index layer.

The chiroptical platform in the simulations is covered with a $5 \mathrm{~nm}$ uniform and isotropic chiral film, whose optical constants are chosen to match the optical properties of Flavin mononucleotide with a surface concentration of $100 \mathrm{ng} / \mathrm{cm}^{2}\left(\omega_{0}=2 \pi c / \lambda_{0}, \lambda_{0}=\right.$ $380 \mathrm{~nm}, \gamma_{\mathrm{c}}=2.5 \cdot 10^{-3} \mathrm{eV}, \beta_{\mathrm{c}}=2.5 \cdot 10^{-9} \mathrm{eV} / \Omega, \Gamma=0.1 \mathrm{eV}$ and $\left.\varepsilon_{\mathrm{c} 0}=1.77\right) .{ }^{9,10}$

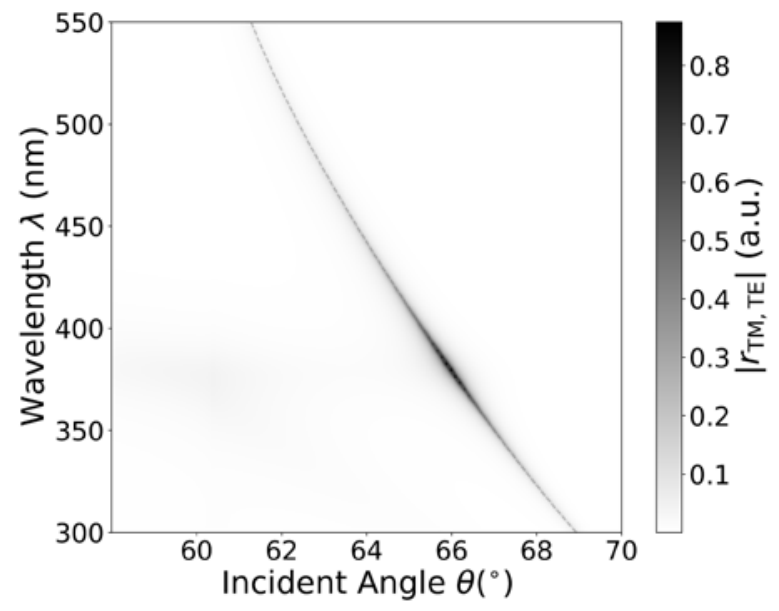

FIGURE 4 Density plot of the $\left|r_{T M, T E}\right|$ term for a $100 \mathrm{ng} / \mathrm{cm}^{2}$ film deposited on the 1DPC platform. The maxima are aligned along the superposition of the TE and TM surface modes, with peak values at the intersection between the dispersion relations and the molecular transition at $\lambda \sim 380 \mathrm{~nm}$.
The reflection maps as a function of the angle of incidence $\theta$ and the wavelength $\lambda$, for both TE and TM illuminations, are reported in Figure 3(a) and Figure 3(b). The total internal reflection onset is clearly visible around $\theta \sim 60^{\circ}$, while the surface modes emerge as a narrow dark band (TE) and a broader dark halo (TM) cutting diagonally through the maps. The mode overlapping is demonstrated in Figure 3(c), which reports the superposition of both reflection maps. The TE and TM modes are nicely superimposed over a broad spectral range from $300 \mathrm{~nm}$ to $500 \mathrm{~nm}$.

Additional understanding is provided by Figure 4 , which reports the $(\theta, \lambda)$ map of the $\left|r_{T M, T E}\right|$ term for the same surface coverage of chiral molecules. Figure 4 distinctly shows a marked maximum along the superposition of the surface modes, offering a strong confirmation that a sizable chiroptical signal emerges with the joint presence of superchiral surface waves and optically active materials at the interface. We remark that, if we substitute the $5 \mathrm{~nm}$ chiral layer with a non-chiral one $\left(\beta_{\mathrm{c}}=0\right),\left|r_{T M, T E}\right|$ becomes identically zero in agreement with the cylindrical symmetry of the sample and the lack of optical activity. ${ }^{26}$

Figure 5 demonstrates the reflectivity dichroism map for the same configuration, further corroborating these conclusions. The map reveals a strong signal aligned with the chiral surface mode. The maximum signal appears at the crossing between the molecule electronic transition (inset of Figure 5) and the chiral modes, reaching values up to $R D \simeq 6$ mdeg (millidegrees: historically, $\mathrm{CD}$ is expressed as ellipticity in mdeg with $\Delta A_{\text {mdeg }} \simeq$ $32980 \cdot \Delta A$ ), with signal enhancements up to $R D / C D_{\text {ref }} \simeq 150$. The inset in Figure 5 further reveals that, by following the dispersion relation of the chiral surface mode in the $(\theta, \lambda)$ plane (light gray dashed line in Figure 5), it is possible to reconstruct the line shape of the original molecular transition. The enhanced signal nevertheless displays a slight broadening of the transition linewidth, which is caused by a modulation of the sensor efficiency introduced by the molecular absorption.

We perform a similar exercise by mapping the ellipticity $\chi$ and the rotation $\psi$ for TE and TM illuminations (see Figure 6). Similarly to what we found for the calculations of the reflectivity dichroism, large signals are only present along the superposition of the dispersion relations. It is also worth noting that the ellipticity map for the TM illumination closely resembles the behavior observed

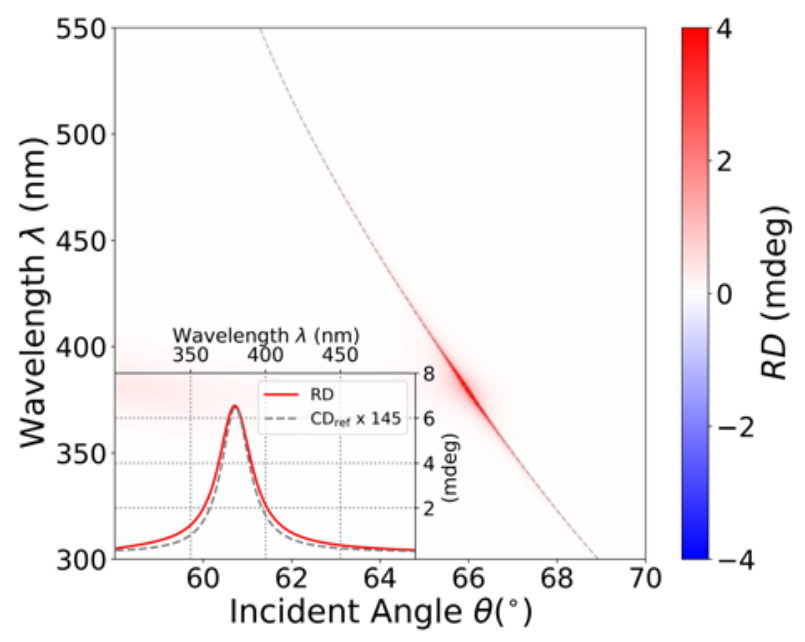

FIGURE 5 Density plot of the modeled $R D$ signal for a $5 \mathrm{~nm}, 100 \mathrm{ng} / \mathrm{cm}^{2}$ molecular layer on top of the 1DPC. The maximum of the $R D$ signal mirrors the behaviour of the $\left|r_{T M, T E}\right|$ term. (inset) Enhanced $R D$ spectrum (red line) retrieved following the dispersion relation (light grey dashed line in the map), compared with a reference CD spectrum for a $100 \mathrm{ng} / \mathrm{cm}^{2}$ molecular film deposited on a glass substrate (dashed line). 

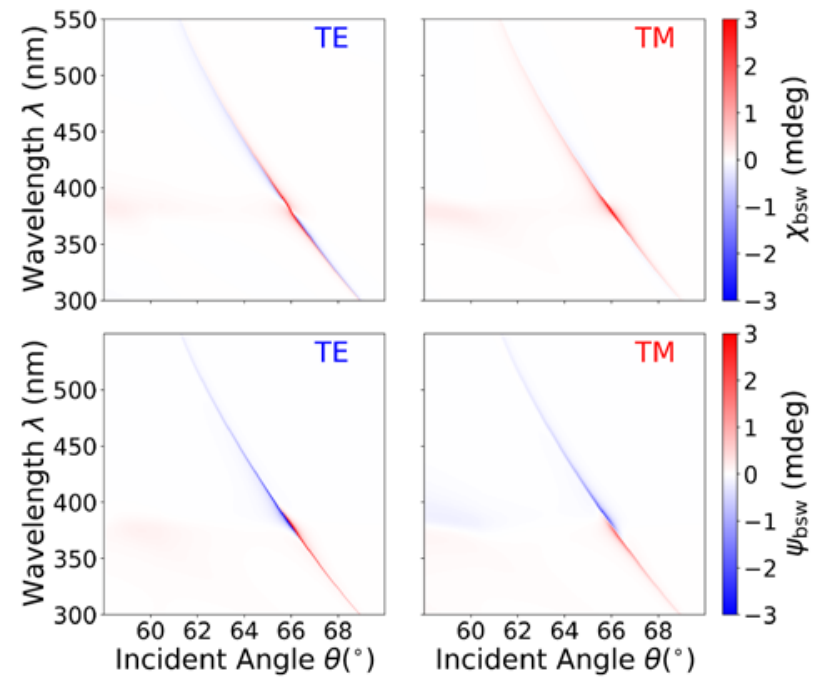

FIGURE 6 Ellipticity (first row) and rotation (second row) density plots for a $100 \mathrm{ng} / \mathrm{cm}^{2}$ film deposited on the 1DPC platform.

for the RD maps. This is readily understood in terms of Equation 9 and Equation 14, where $\left|r_{T E, T E}\right|<\left|r_{T M, T M}\right|$ (see Figure 3) leads to the more prominent contribution of the TM polarization term in the $\mathrm{RD}$ signal. This similarity is indeed lost in the case of the TE ellipticity map. For the anisotropic superchiral platform under investigation, therefore, care must be taken since the standard linear relation between $C D$ and $\chi$ that is encountered in the analysis of chiral molecules in an isotropic background breaks down. We finally underline that both rotation density plots display a positive signal at smaller wavelengths, which then changes to a negative value around $\lambda \simeq 380 \mathrm{~nm}$, at correspondence with the energy of the electronic transition. This behavior recalls the characteristic sigmoidal shape which is usually observed in rotation spectra at resonance.

To complete the investigation, we study the response of the 1DPC sensing platform as the molecule surface concentration is varied by one order of magnitude from $100 \mathrm{ng} / \mathrm{cm}^{2}$ to $1 \mu \mathrm{g} / \mathrm{cm}^{2}$. We do this by first varying the volumetric concentration for a fixed layer thickness of $50 \mathrm{~nm}$ (which is achieved by tuning the values of $\gamma_{\mathrm{c}}$ and $\beta_{\mathrm{c}}$ ) and then by varying the molecular layer thickness from $5 \mathrm{~nm}$ to $50 \mathrm{~nm}$ while keeping the volumetric concentration constant. Figure 7(a) reports the effective enhancement spectra where, as done before, the RD signal is retrieved along the superposition of the dispersion relations (dashed line in Figure 5). It is immediately apparent how larger surface concentrations result in lower enhancements. This is easily explained in terms of the additional damping introduced by the molecules at the 1DPC surface, which leads to weaker and broader surface modes and likewise to smaller superchiral fields. The described effect is more prominent at correspondence with the molecular electronic transition, where the losses are inherently larger, which leads to the slight spectral modulation observed in the inset of Figure 5. The same reduction in enhancement efficiency occurs in Figure $7(b)$, where the surface concentration is instead varied by changing the molecular film thickness. In this case, the variation observed between the different enhancement spectra is also influenced by the exponential decay of BSWs away from the 1DPC surface.

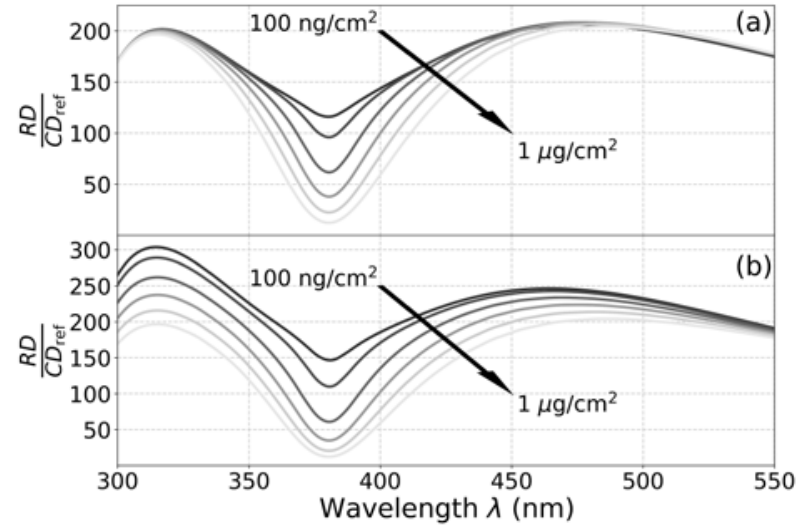

FIGURE $7 R D / C D_{\text {ref }}$ enhancement retrieved along the superposition of the two dispersion relations (light grey dashed line of Fig.4). (a) Fixed 50 nm thickness and varying surface concentration from $100 \mathrm{ng} / \mathrm{cm}^{2}$ to $1 \mu \mathrm{g} / \mathrm{cm}^{2}$. (b) Varying surface concentration (and thickness) from $100 \mathrm{ng} / \mathrm{cm}^{2}$ to $1 \mu \mathrm{g} / \mathrm{cm}^{2}$ (5 nm to $50 \mathrm{~nm}$ ).

\section{Conclusions}

In conclusion, we studied the chiroptical response of a 1DPC supporting superchiral surface waves coupled with a uniform and isotropic optically active thin film deposited on its surface. We introduced a simple formalism that, starting from the Fresnel reflection matrix, allows us to retrieve analytical expressions for all the relevant chiroptical quantities associated with the response of the 1DPC covered with a layer of chiral molecules. The sensing platform provides chiral signal enhancements of more than two orders of magnitude over a broad spectral range. Finally, we analyzed the sensor performance as a function of the analyte surface concentration, highlighting that excessive concentrations can lead to a degradation in the sensor performance.

\section{Acknowledgements}

The research leading to these results has received funding from the Italian Ministry of Education, Universities and Research (MIUR) through the PRIN 2015 program (Project No. 2015FSHNCB "Plasmon-enhanced vibrational circular dichroism")

\section{REFERENCES AND NOTES}

\section{Journal}

1. Barron, L. D. Molecular light scattering and optical activity. (Cambridge University Press, 2004)

2. Hendry, E. et al. Ultrasensitive detection and characterization of biomolecules using superchiral fields. Nat. Nanotechnol. 5, 783-787 (2010).

3. Development of New Stereoisomeric Drugs. (U.S. Food and Drug Administration, 1992)

4. Chiral technology - A global strategic business report. (Global Industry Analysts, Inc., 2012).

5. Tang, Y. \& Cohen, A. E. Optical Chirality and Its Interaction with Matter. Phys. Rev. Lett. 104, 163901 (2010)

6. Tang, Y. \& Cohen, A. E. Enhanced Enantioselectivity in Excitation of Chiral Molecules by Superchiral Light. Science 332, 333-336 (2011). 
7. Schäferling, M., Yin, X., Engheta, N. \& Giessen, H. Helical Plasmonic Nanostructures as Prototypical Chiral Near-Field Sources. ACS Photonics 1, 530-537 (2014).

8. Schäferling, M., Yin, X. \& Giessen, H. Formation of chiral fields in a symmetric environment. Opt. Express 20, 26326-26336 (2012).

9. Nesterov, M. L., Yin, X., Schäferling, M., Giessen, H. \& Weiss, T. The Role of Plasmon-Generated Near Fields for Enhanced Circular Dichroism Spectroscopy. ACS Photonics 3, 578-583 (2016).

10. Abdulrahman, N. A. et al. Induced Chirality through Electromagnetic Coupling between Chiral Molecular Layers and Plasmonic Nanostructures. Nano Lett. 12, 977-983 (2012).

11. Schäferling, M., Engheta, N., Giessen, H. \& Weiss, T. Reducing the Complexity: Enantioselective Chiral Near-Fields by Diagonal Slit and Mirror Configuration. ACS Photonics 3, 1076-1084 (2016).

12. Govorov, A. O. \& Fan, Z. Theory of Chiral Plasmonic Nanostructures Comprising Metal Nanocrystals and Chiral Molecular Media. ChemPhysChem 13, 2551-2560 (2012).

13. Hendry, E., Mikhaylovskiy, R. V., Barron, L. D., Kadodwala, M. \& Davis, T. J. Chiral Electromagnetic Fields Generated by Arrays of Nanoslits. Nano Lett. 12, 3640-3644 (2012).

14. Schäferling, M., Dregely, D., Hentschel, M. \& Giessen, H. Tailoring Enhanced Optical Chirality: Design Principles for Chiral Plasmonic Nanostructures. Phys. Rev. X 2, 031010 (2012)

15. Valev, V. K., Baumberg, J. J., Sibilia, C. \& Verbiest, T. Plasmonic Nanostructures: Chirality and Chiroptical Effects in Plasmonic Nanostructures: Fundamentals, Recent Progress, and Outlook (Adv. Mater. 18/2013). Adv. Mater. 25, 2509-2509 (2013).
16. Lu, F. et al. Discrete Nanocubes as Plasmonic Reporters of Molecular Chirality. Nano Lett. 13, 3145-3151 (2013).

17. Frank, B. et al. Large-Area 3D Chiral Plasmonic Structures. ACS Nano 7, 6321-6329 (2013).

18. Liu, Y., Wang, R. \& Zhang, X. Giant circular dichroism enhancement and chiroptical illusion in hybrid molecule-plasmonic nanostructures. Opt. Express 22, 4357-4370 (2014).

19. Valev, V. K. et al. Nonlinear Superchiral Meta - Surfaces: Tuning Chirality and Disentangling Non - Reciprocity at the Nanoscale. Adv. Mater. 26, 4074-4081 (2014).

20. Finazzi, M., Biagioni, P., Celebrano, M. \& Duò, L. Quasistatic limit for plasmon-enhanced optical chirality. Phys. Rev. B 91, 195427 (2015).

21. Knight, M. W. et al. Aluminum for Plasmonics. ACS Nano 8, 834-840 (2014).

22. Pellegrini, G., Finazzi, M., Celebrano, M., Duò, L. \& Biagioni, P. Chiral surface waves for enhanced circular dichroism. Phys. Rev. B 95, 241402 (2017).

23. Yeh, P., Yariv, A. \& Hong, C.-S. Electromagnetic propagation in periodic stratified media. I. General theory. J. Opt. Soc. Am. 67, 423-438 (1977).

24. Sinibaldi, A. et al. Combining label-free and fluorescence operation of Bloch surface wave optical sensors. Opt. Lett. 39, 2947-2950 (2014).

25. Jaggard, D. L. \& Sun, X. Theory of chiral multilayers. J. Opt. Soc. Am. A 9, 804 (1992).

26. Arteaga, O. Useful Mueller matrix symmetries for ellipsometry. Thin Solid Films 571, 584-588 (2014). 